\title{
Epidemiology of bovine respiratory disease (BRD) in preweaned calves on California dairies: The BRD 10K study
}

\author{
S. A. Dubrovsky, ${ }^{1}$ A. L. Van Eenennaam, ${ }^{1}$ B. M. Karle, ${ }^{2}$ P. V. Rossitto, ${ }^{3}$ T. W. Lehenbauer,${ }^{3,4}$ and S. S. Aly ${ }^{3,4 *}$ \\ ${ }^{1}$ Department of Animal Science, University of California, Davis 95616 \\ ${ }^{2}$ Cooperative Extension, Division of Agriculture and Natural Resources, University of California, Orland 95963 \\ ${ }^{3}$ Veterinary Medicine Teaching and Research Center, School of Veterinary Medicine, University of California, Davis, Tulare 93274 \\ ${ }^{4}$ Department of Population Health and Reproduction, School of Veterinary Medicine, University of California, Davis 95616
}

\section{ABSTRACT}

Bovine respiratory disease (BRD) is one of the leading causes of death in dairy heifers. The objective of this prospective cohort study was to characterize the epidemiology of BRD in preweaned dairy calves and to identify management practices associated with decreased risk of BRD. Dairies were chosen for the study based on management practices, location, size, and willingness to participate. A total of 6 dairies, ranging in size from 700 to 3,200 milking cows, in 6 counties across California's Central Valley, were enrolled in the study for at least 1 year. A total of 11,945 calves were born on the study dairies and followed until weaning. Incidence of BRD was estimated using treatment records. Trained study personnel performed comprehensive calf management surveys and estimated BRD prevalence on every dairy at least once every season. A shared frailty model was used to model the associations between management practices and BRD hazard. The final models included data from complete records of 11,470 calves. The overall BRD study period prevalence across the study herds was $22.8 \%$. The mean BRD incidence density rate on all the study dairies was 0.17 BRD cases [95\% confidence interval (CI) $=0.16-1.74$ ] per calf-month at risk. The shared frailty model identified that feeding only waste or saleable milk (compared with use of milk replacer), feeding over $3.8 \mathrm{~L}$ of milk per day to calves under $21 \mathrm{~d}$ of age, frequent changing of maternity pen bedding, and administration of modified live or killed BRD vaccines to dams before calving significantly reduced the risk of BRD. Risk factors for BRD included housing calves in wooden hutches with metal roofs, compared with all-wood hutches, twin births, and perception of dust occurring "regularly," as

Received March 20, 2018.

Accepted April 9, 2019.

*Corresponding author: saly@ucdavis.edu reported by calf managers, compared with a perception of "no dust" in the calf-raising area. All 4 seasons were analyzed, and both summer (hazard ratio $=1.15 ; 95 \%$ $\mathrm{CI}=1.01$ to 1.32 ) and spring (hazard ratio $=1.26 ; 95 \%$ $\mathrm{CI}=1.11$ to 1.44 ) were associated with a higher risk of BRD compared with winter. The current longitudinal study identified specific housing and feeding practices that could be modified to decrease risk of BRD. In addition, season was observed to have a strong effect on calves' risk of developing BRD on California dairies. Key words: bovine respiratory disease, dairy calf, epidemiology, pneumonia

\section{INTRODUCTION}

One of the leading causes of morbidity in preweaned dairy calves in the United States is bovine respiratory disease (BRD; USDA, 2012). Bovine respiratory disease is multifactorial and can be caused by the interaction of pathogenic bacteria and viruses, leading to a variety of clinical signs, which complicates the development of effective treatment protocols and prevention practices. Despite advancements in treatments and changes in management practices, the national herdlevel incidence of BRD in dairy calves (from birth to weaning) has remained constant at around $22 \%$, and BRD continues to be the cause of $30 \%$ of all calf mortalities (Gardner et al., 1990; Chigerwe et al., 2015).

Decreasing the incidence of BRD is of considerable importance, as several studies have shown that, in addition to the costs associated with treating sick calves, diagnosis of BRD during calfhood is associated with a lifetime productivity decrease in cattle (Warnick et al., 1994, 1997). The decrease in productivity occurs due to a variety of factors, including increased likelihood of mortality, dystocia, culling before the first calving, and lower milk yields during first lactation (Schaffer et al., 2016).

Unlike BRD research reports from Canada, the eastern United States, and Europe, there have been 
no comprehensive longitudinal studies characterizing the epidemiology of BRD in preweaned calves in the state of California since 1990 (Curtis et al., 1988; Gardner et al., 1990; Virtala et al., 1996; Svensson et al., 2003). California has mild winters and extremely hot and dry summers. Hence, studies conducted in other regions, especially those with harsher winters, identify risk factors that are often exclusive to colder climates, related to indoor housing and ventilation, rendering them not applicable to California (Lago et al., 2006). For example, indoor housing of calves during winter in cold climate regions commonly involves group housing, which has been associated with the greatest risk of BRD compared with other housing types (Losinger and Heinrichs, 1996; Svensson et al., 2003). In contrast, the mild winters of California allow for outdoor housing of calves year-round, with special consideration of nutrient requirements during cooler months. Similarly, the dairy industry in the northeastern United States has smaller herds (Pithua et al., 2009), limiting the application of findings for that region among the larger Californian herds (CDFA, 2016).

The objectives of the current study were to estimate seasonal variations in BRD incidence density rates (IDR) and to identify management practices or factors associated with reduced risk of BRD in preweaned dairy calves in California. Findings from the current study will be used to develop an evidence-based risk assessment tool for use by veterinarians and dairy managers to reduce the incidence of $\mathrm{BRD}$ in preweaned dairy calves.

\section{MATERIALS AND METHODS}

This study was approved by the Institutional Animal Use and Care Committee at the University of California, Davis (Protocol \#17496).

\section{Study Herds Selection}

Study herds were selected based on size, location, diversity of management practices, calf record keeping, and owner willingness to participate. Between March and August of 2015, 6 herds were enrolled in the study. The study herds were located in Northern California (2 dairies) and both the northern San Joaquin Valley (3 dairies) and the greater Southern California region (1 dairy), to represent the state's 3 distinct dairy management systems and their characteristics (Figure 1). The criteria considered when selecting the study dairies included location and herd size; specifically, the study included 2 small herds (under 1,000 milking cows), 3 medium-sized herds (over 1,000 milking cows

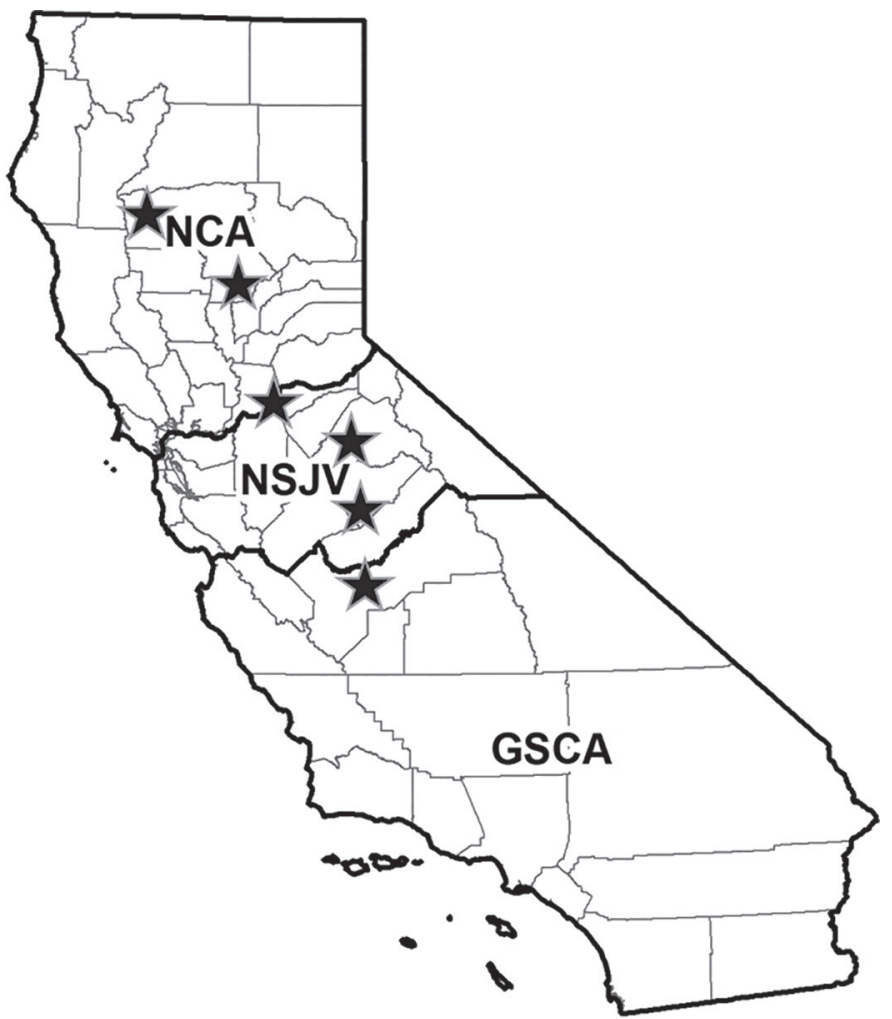

Figure 1. California map identifying Northern California (NCA), the northern San Joaquin Valley (NSJV), and the greater Southern California region (GSCA), which includes the southern half of the San Joaquin Valley. Also identified in the map (stars) are the counties where dairies were enrolled in our longitudinal study of bovine respiratory disease in preweaned dairy calves.

but fewer than 3,000), and 1 large herd (over 4,000 milking cows). In addition, dairies were also selected to include a variety of calf housing types as well as other management practices, including type of milk fed to calves and colostrum management (Love et al., 2016a). Of the study dairies, 4 had previously participated in a state-wide survey of management practices pertaining to BRD (Love et al., 2016a) and the BRD 100, a BRD prevalence study (Karle et al., 2019; Maier et al., 2019). The current article presents findings related to BRD morbidity; findings related to BRD-specific mortality in California are presented in a different article ( $\mathrm{Du}-$ brovsky et al., 2019).

\section{Calf Enrollment and Exclusion Criteria}

At commencement of the study, all preweaned calves without history of treatment for BRD on each study dairy were enrolled. Subsequently, calves were enrolled at birth and followed until weaning. Calves that were diagnosed with BRD, that were sold, or that died be- 
fore weaning were assigned the date of those events as their exit date. Male calves born on the study dairies and sold within $2 \mathrm{~d}$ of birth were not enrolled in the study. Any calves, male or female, sold more than $2 \mathrm{~d}$ after birth were included in the study. Any calves that died within $24 \mathrm{~h}$ of birth, or that were stillborn, were not enrolled in the study.

\section{Treatment Records}

The study dairies' calf health staff reported use of observations on cough, nasal discharge, increased respiratory rate, ocular discharge, and depression/fatigue as clinical signs to diagnose BRD. For the current study, the treatment crews were trained by study personnel and asked to complete a treatment card for each treated BRD case. Treatment cards included space for information on calf identification number (ID), breed, date of birth, sex, date of treatment, medications administered, and a checklist of clinical signs. The clinical signs checklist included those from the California BRD scoring system: eye discharge, nasal discharge, head tilt/ear droop, cough, increased respiration rate, and temperature (Love et al., 2014). Calves were defined as having BRD when the dairy staff made the decision to treat the calf for BRD; at this point, staff filled out the treatment cards, including the clinical signs used to conclude that the calf had BRD. Treatment cards were printed on weatherproof material adhered to polypropylene tags measuring 10 by $10 \mathrm{~cm}$ and affixed to calf hutches using staples or adhesive tape. Most dairies attached the cards to the hutches once a calf became a case and returned the cards to study personnel when the calf was weaned. On one dairy, calves were able to reach around to chew on the cards; hence, cards were not affixed to the hutches at that site and instead were kept by the calf caretaker. If a calf died before weaning, calf caretakers were instructed to note the date of death on the treatment card and return the treatment cards for dead calves to the study personnel.

\section{Data Collection}

A comprehensive management questionnaire based on a statewide BRD survey was administered at enrollment and again every 2 to 4 mo for the duration of the study, to incorporate seasonal management changes (Love et al., 2016a). At each assessment, trained personnel interviewed the dairy calf manager or owner and asked questions about herd demographics, calving practices, calf housing, colostrum management and feeding, feeding programs for calves, respiratory disease vaccinations, and dust management practices. Questions in the first assessment referred to the previous 3 mo on the study dairy. Subsequent questionnaires referred to the period since the previous assessment.

In addition, at each visit, a subset (random sample) of calves on each dairy was selected and scored by researchers using the California BRD scoring system to obtain a BRD prevalence estimate, defined as the proportion of calves identified as BRD cases over the total number of calves sampled. The prevalence estimate had a bound on error of estimation $(B)$ assumed to be $10 \%$, and was estimated based on a sample size $(n)$ proportional to the size of the preweaned calf herd [Eq. 1]:

$$
n=\frac{N p q}{(N-1) D+p q},
$$

where $q=1-p$, and $D=B^{2} / 4$, and where $p$ is the population prevalence, assumed to be $25 \%$ based on the study investigators' previous experience of the prevalence of BRD between birth and weaning on California dairies (Gardner et al., 1990; Scheaffer et al., 2006), and $N$ is the total number of calves in the herd. This estimate was intended to provide a snapshot of BRD prevalence on the dairies, to allow comparison with studies that only report prevalence estimates, and was not incorporated in the shared frailty model for incidence presented elsewhere.

Study personnel visited the study herds every 2 to 3 weeks for the duration of the study to provide outreach and education to the dairy staff, observe calf feedings and treatments, observe disease recordings, assign treatment cards to calves, and collect treatment cards from weaned calves' hutches and calf caretakers. In addition, during visits, investigators observed management practices and reconciled the observed practices with questionnaire responses, and recorded hutch type for newborn calves on dairies with multiple hutch types.

\section{Data Housing and Relational Database}

The study data were stored in a relational database (Access, Microsoft Corporation, Redmond, WA). Dairy survey responses were linked to their respective individual calf records (calf ID, birthdate, sex, events of interest, twinning status). Specifically, survey responses were time matched to calf records using calf birthdate and the time interval the survey covered. Calf ID was linked to events of interest (date of death, weaning date, BRD diagnosis date, date sold). Calf ID was also linked to BRD case records (symptoms listed, date of treatment, and treatment abbreviations). Treatments were linked into a case records table that contained 
all of the treatment protocols for BRD in preweaned calves.

\section{Descriptive Statistics}

Measures of central tendency and uncertainty (mean and standard deviation, respectively) were computed for variables related to the herd (milking cow herd size, age at first calving, BRD prevalence, times per month maternity pen bedding was changed), calf management (preweaned calf herd size, weaning age, percent colostrum from uniparous dams), and calf feeding (volume and type of milk fed - saleable, waste, or milk replacer).

Clinical signs reported on the treatment card were used to generate a diagnosis of BRD based on the California BRD scoring system, called the Scoring System Diagnosis (SSD). Separately, dairy staff were allowed to identify and treat BRD cases based on their on-farm criteria. Agreement between the California BRD scoring system and calf caretakers' diagnoses was measured. To obtain a quantitative measure of agreement between the SSD and the calf caretakers' diagnoses of BRD, the 2 different diagnostic methods were evaluated for interrater agreement by estimating the kappa statistic (Cohen, 1960):

$$
\hat{k}=\frac{\left(p_{0}-p_{e}\right)}{\left(1-p_{e}\right)},
$$

where $\hat{k}$ is the kappa statistic, $p_{0}$ is the observed proportion of agreement between the 2 BRD diagnostic methods, and $p_{e}$ is the expected proportion of agreement between the 2 methods.

\section{Cumulative Incidence and IDR}

Cumulative incidence and IDR of BRD were estimated for all study calves, stratified by herd, twin birth status, season, and calf sex. Cumulative incidence was estimated as the proportion of new cases of BRD that occurred between enrollment and the end of the followup period. The IDR was calculated for each of the 2 methods of BRD diagnosis: one determined by the calf caretakers and the other by using the SSD as described previously, using Equation 3:

$$
\text { Incidence density rate }=\frac{\text { No. of BRD cases }}{\text { Sum of calf-days at risk }} .[3]
$$

Days at risk for each calf included every day from birth to the event of interest (BRD diagnosis) or censoring (weaning, death, or sale). Calves were at risk for $51 \mathrm{~d}$ (birth to weaning) on some dairies, although calves on other dairies were at risk for $79 \mathrm{~d}$. The IDR accounted for such differences by referencing the number of cases (numerator) to the number of calf-days at risk (denominator). Season was categorized as winter (December 1 through February 28), spring (March 1 through May 31), summer (June 1 through August 31), and fall (September 1 through November 30). Six calves did not have a designation as twin or single calves and were assumed to be single-calf births. The sex of 20 calves was not recorded; however, they were assumed to be female because they were born on dairies where only female calves were assigned identification numbers.

\section{Shared Frailty Model}

Preweaned calves on the participating dairies at the beginning of the study had the study start date as their enrollment date. Calves born after the study began had their birth dates as their enrollment dates. Each calf in the study was followed from its enrollment date to an occurrence of the event of interest or censoring. The event of interest for survival analysis was a BRD diagnosis based on calf caretaker recording of clinical signs in treatment records of study calves. Censoring otherwise occurred when a calf was weaned, was sold, or died before weaning. All enrolled calves exited the study at BRD diagnosis or censoring. Calf ID was recorded along with hutch types throughout the study on the dairies (with occasional gaps due to study personnel schedules), to match calves with hutch types, as many dairies had more than one hutch type. Any empty hutches were noted and checked against dairy records on deceased or sold calves.

A frailty model was used to account for dependence among calves born on the same dairy acting on the hazard function. A shared gamma-distributed frailty model was run, with the shared frailty variable specified as dairy, to adjust for herd effect, using this formula:

$$
h_{i j}(t)=\alpha_{j} h_{0}(t) \exp \left(\beta X_{i j}\right),
$$

where $h_{i j}$ is the conditional hazard function for the $i$ th calf in the $j$ th herd (Cohen, 1960; Kleinbaum and Klein, 2005). The baseline hazard $h_{0}(t)$ at time $t$ was multiplied by $\alpha_{j}$, the model frailty, where all calves born on the same dairy had the same $\alpha_{j}$. The $\beta X_{i j}$ expression represents the subjects' covariates, including herd and calf demographics and variables related to housing, colostrum management, feeding, vaccinations, and dust management.

Continuous variables were assessed for outliers, identified using the Tukey test for outliers; categorical independent variables were coded as dichotomous for 
either absence or presence of a factor, or were specified with dummy variables, with the referent level being absence or the lowest level of a risk factor based on post hoc analysis, respectively. Each variable was first examined in a univariate shared frailty model, and considered statistically significant if $P<0.05$, before being included in the full multivariate model. If a continuous variable was not significant in a univariate model, the variable was subsequently examined with frequency plots and categorized into approximately equal categories and then analyzed as a categorical variable in a univariate model. A manual forward model-building algorithm was applied, with variables related to herd and calf demographics entered first, followed by those related to housing, colostrum management, feeding, vaccinations, and finally dust-related variables. Interactions between the dairies and all significant variables were investigated. Confounding was assessed using the method of change in estimates, contrasting models with and without potential confounders. A change greater than $20 \%$ in model estimates resulted in inclusion of the potential confounding variable in the final model. A $5 \%$ level of significance was considered for statistically significant differences.

Two different methods were used to assess model goodness of fit. First, the likelihood ratio test was used to compare competing nested models first with a full model that included the variable of interest and then with a reduced model without the variable of interest (Greene, 2012). Second, a model with a lower Akaike information criterion estimate was denoted as a better fit (Akaike, 1998).

Dam- and Calf-Related Variables. The effects of twinning, being born to dams that had undergone in vitro fertilization or embryo transfer, season, and number of times maternity pen bedding was changed per month were included in the dam- and calf-related variables. Breed was also explored as a predictor, as were 2-way interaction terms for breed and dairy, between milk replacer use and type of milk fed (saleable or waste), and between type of milk fed and whether the milk was pasteurized.

Housing-Related Variables. Housing variables included additional shade structures, hutch type, whether calves in adjacent hutches could touch, and whether hutches were elevated and a flush system used to clean manure. Several variables relating to dust in the calf area were examined: road surfaces around the hutches, and a variable based on whether dust was observed by the manager in the calf-raising area.

Colostrum Management Variables. Variables related to colostrum fed and its absorption included whether calves were fed pooled colostrum, colostrum quality as assessed using a colostrometer, and colostrum supplementation with colostrum replacer. Percentage of colostrum fed from first-calf heifers, evaluation of calves' serum total protein as an indicator of failed passive transfer of immunity by the dairy, volume of colostrum fed in the first $12 \mathrm{~h}$, and colostrum feeding method were also explored as predictors for hazard of BRD. Variables related to colostrum handling included heat treatment of colostrum, addition of a preservative, number of hours colostrum was stored before feeding, type of colostrum storage container, and colostrum storage temperature. Two-way interactions between supplementation with colostrum replacer and source of colostrum were also tested.

Calf Feeding-Related Variables. The order in which calves were fed in relation to age and health status was investigated. Other variables related to the milk fed to calves included the type of milk fed, whether calves under $21 \mathrm{~d}$ were fed waste milk, whether milk was pasteurized and whether it was tested for bacterial content before or after pasteurization (or both), use of milk replacer, volume of milk fed per feeding (a categorical variable), and antibiotics added to milk fed. Variables for method of feeding of milk (bucket or bottle), whether dairy staff reduced milk feedings to condition the calves before weaning, and intervals between feedings were examined. We also explored whether the youngest calves were offered water and whether water containers were filled within an hour of feeding milk.

Immunization-Related Variables. Modified live and killed vaccines used on the study dairies included antigens for bovine viral diarrhea virus (type 1 and 2 ), infectious bovine rhinotracheitis, parainfluenza 3, and bovine respiratory syncytial virus. The intranasal vaccines used on the study dairies had similar compositions to one another, with the exception of that for bovine viral diarrhea. Whether calves were vaccinated using intranasal or injectable vaccines before weaning was explored as a predictor of BRD only if vaccination occurred at least $15 \mathrm{~d}$ before weaning, to allow time for development of an immune response. In addition, use of a killed or modified live vaccine in pregnant dams was also explored, along with interaction terms with the volume of colostrum fed in the first $12 \mathrm{~h}$ after birth. Similarly, an interaction term between vaccines administered to cows and the use of colostrum replacer was also explored, as previous studies have found an association between the vaccines administered to pregnant cows and heifers and the quality of colostrum (Hodgins and Shewen, 1994). 


\section{RESULTS}

\section{Descriptive Statistics}

A total of 11,945 calves, $68.5 \%$ female and $31.5 \%$ male, were enrolled between March 2015 and July 2016 on the 6 study dairies. The study herds are summarized in Table 1. Dairy 1 was primarily Holstein, and dairies 2, 3, and 4 were exclusively Holstein. Dairy 5 was primarily a Jersey herd (less than $10 \%$ Jersey $\times$ Limousin-cross calves). The study calves had a mean prevalence of preweaning BRD of $8.19 \%$ (2.93\% to $12.62 \%$ ), based on diagnosis with the California BRD scoring system over the entire study period and across all the study dairies. Tables 2 and 3 summarize the study dairies' calving and colostrum management, respectively. In addition to raising heifer calves, dairies $1,2,4$, and 5 raised male calves, whereas the remaining dairies sold all their male calves within a few days of birth. The calf populations for dairies 2 and 4 included approximately $6 \%$ and $4.5 \%$ male calves, respectively. Dairies 1 and 5 bred and sold bull calves for their genetic traits and had approximately $46 \%$ and $15 \%$ male calves, respectively. Table 4 summarizes calf housing practices on the study dairies, and Table 5 summarizes their feeding practices. Two dairies implemented changes in vaccination protocols during the course of the study period. Starting in March 2015, calves enrolled on dairy 1 were born to dams who had received 1 dose of modified live respiratory vaccine, whereas calves enrolled from June 2015 through March 2016 were born to dams not vaccinated with any modified live vaccines. Finally, calves enrolled on dairy 1 between March and June 2016 were born to dams that received 1 modified live vaccine dose. In addition, dairy 1 calves enrolled from March 2015 through October 2015 were born to dams vaccinated with 1 killed respiratory vaccine dose; no other calves were born to dams vaccinated with killed respiratory vaccines on that study dairy. Dairy 2 calves enrolled from March 2015 through April 2016 were born to dams that had received 1 killed respiratory vaccine dose, whereas those enrolled from April to June 2016 were born to dams that were vaccinated with 2 killed respiratory vaccine doses during pregnancy. As a result, approximately $63.5 \%$ and $69.9 \%$ of the study calves were from dairies that implemented changes during the study in their dams' modified live or killed vaccine protocols, respectively.

\section{Cumulative Incidence and Incidence Density Rates}

Interrater agreement between SSD using the California BRD scoring system and BRD cases identified by calf caretakers was $0.83(\mathrm{SE}=0.01)$, as estimated

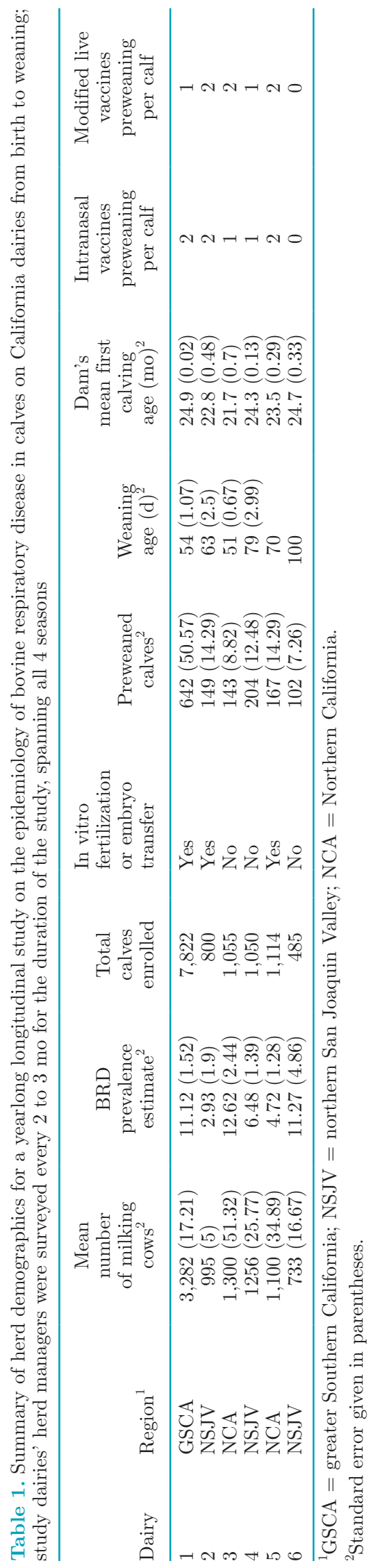

Journal of Dairy Science Vol. 102 No. 8, 2019 
DUBROVSKY ET AL.
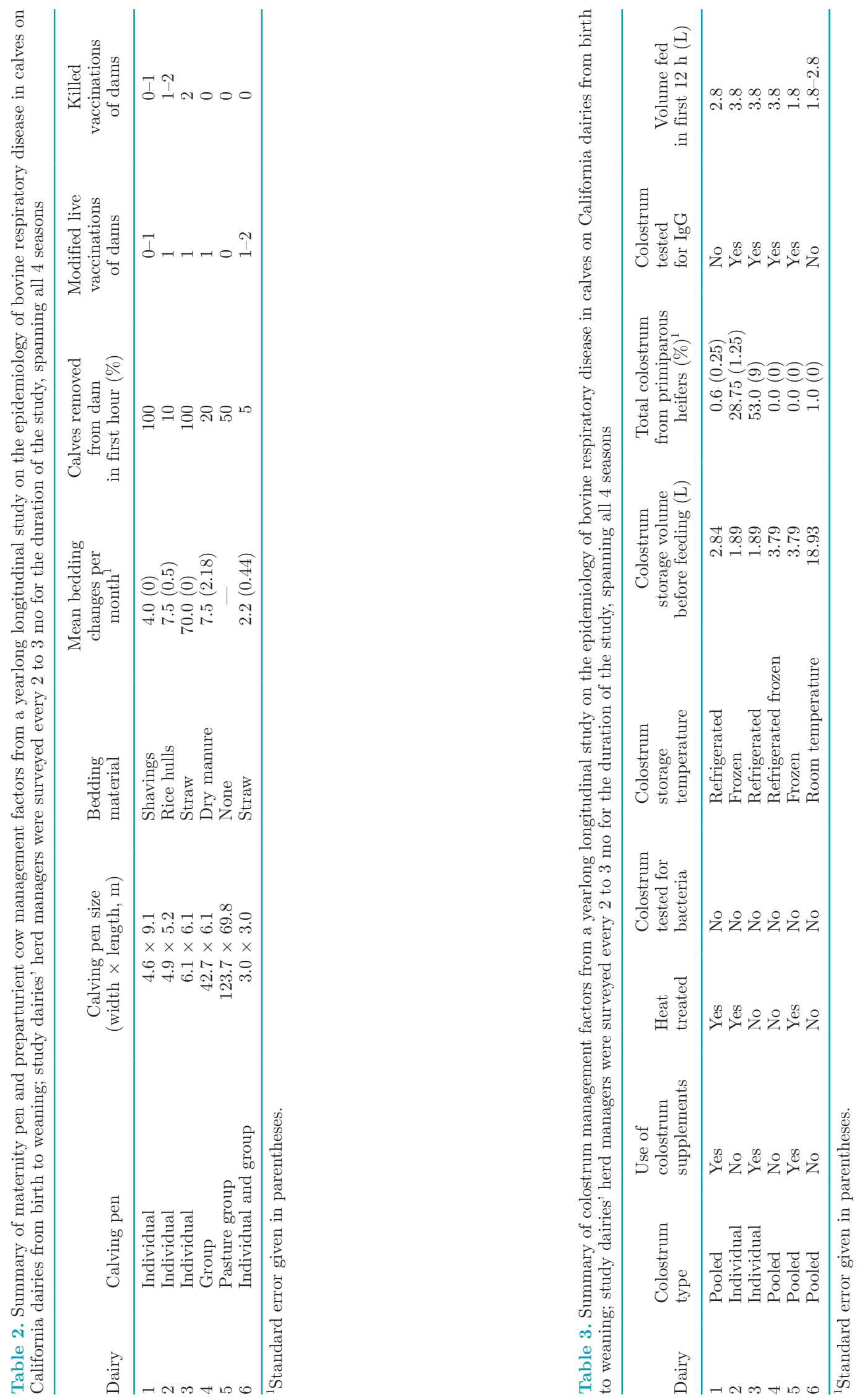
BRD EPIDEMIOLOGY IN CALIFORNIA DAIRY CALVES
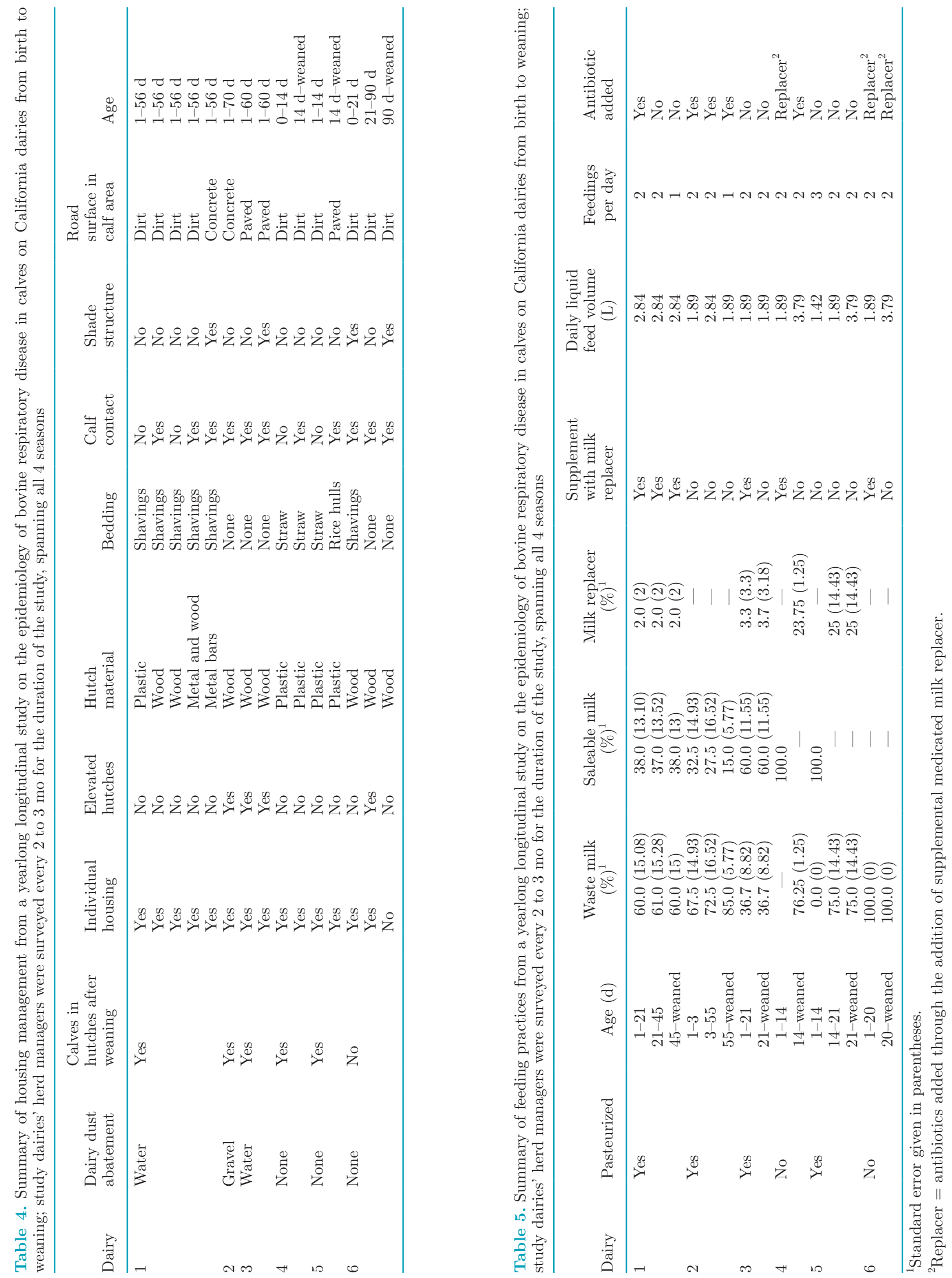
using the kappa statistic, which can be interpreted as "excellent agreement" (kappa $\geq 0.75$; Fleiss et al., 2003). Dairy 6 was excluded from the survival analysis due to noncompliance with study protocols in reporting clinical signs on treatment cards, and as a result only 11,470 calves from the remaining 5 dairies were used in the analysis. Estimates for cumulative incidence and IDR on the study dairies using treatment records and SSD are summarized in Table 6 . The season with the highest BRD IDR was spring at 0.21 cases per calfmonth (95\% CI: $0.20,0.23)$, followed by summer at 0.17 cases per calf-month $(95 \%$ CI: $0.15,0.18)$, fall at 0.14 cases per calf-month (95\% CI: $0.13,0.16)$, and finally winter at 0.14 cases per calf-month $(95 \%$ CI: 0.13 , 0.15). The BRD IDR estimates alternatively can be interpreted as the rate at which cases occurred in the different seasons on the study dairies, to the magnitude of $21,17,14$, and 14 cases of BRD for every 100 calves raised from birth to $30 \mathrm{~d}$ of age in spring, summer, fall, and winter, respectively.

The IDR of BRD among the study's $652(5.7 \%)$ twin calves was 0.22 cases per month $(95 \% \mathrm{CI}=0.19,0.25)$, compared with 0.16 cases per month $(95 \% \mathrm{CI}=0.16$, $0.17)$ among singletons. The cohort of male calves on the study dairies comprised 3,762 (33\%) calves, with a BRD IDR of 0.20 cases per month $(95 \% \mathrm{CI}=0.19$, $0.22)$, compared with 0.16 cases per month $(95 \% \mathrm{CI}=$ $0.15,0.16)$ for the female cohort.

\section{Shared Frailty Model}

Data from 11,300 calves from 5 dairies were used in the shared frailty model. The final shared frailty model is presented in Table 7. Theta, which provided an estimate of the variance between dairies, was significant $(P<0.001)$. The univariate model for the association between modified live vaccination of dams and BRD in dairy 1 calves showed a higher hazard for BRD. However, Kaplan-Meier curves for dairy 1, comparing survival rates for calves of vaccinated dams with those of unvaccinated dams, showed a reduction in hazard of BRD after $50 \mathrm{~d}$ of age. Further exploration showed that age at first enrollment was higher for calves from modified live vaccinated dams than for calves from unvaccinated dams (mean $8.9 \mathrm{~d}$ versus $0 \mathrm{~d}$ of age at enrollment, respectively). More older calves from vaccinated dams were enrolled (from dairy 1 ) than younger calves from unvaccinated dams (from the remaining study dairies) at the start of the study, leading to the mixing of the effect of age and the effect of modified live vaccination of dams. Hence, an indicator variable was created for calves over $50 \mathrm{~d}$ of age in dairy 1 and introduced into the model, whereby confounding was confirmed, as indicated by a change in the magnitude

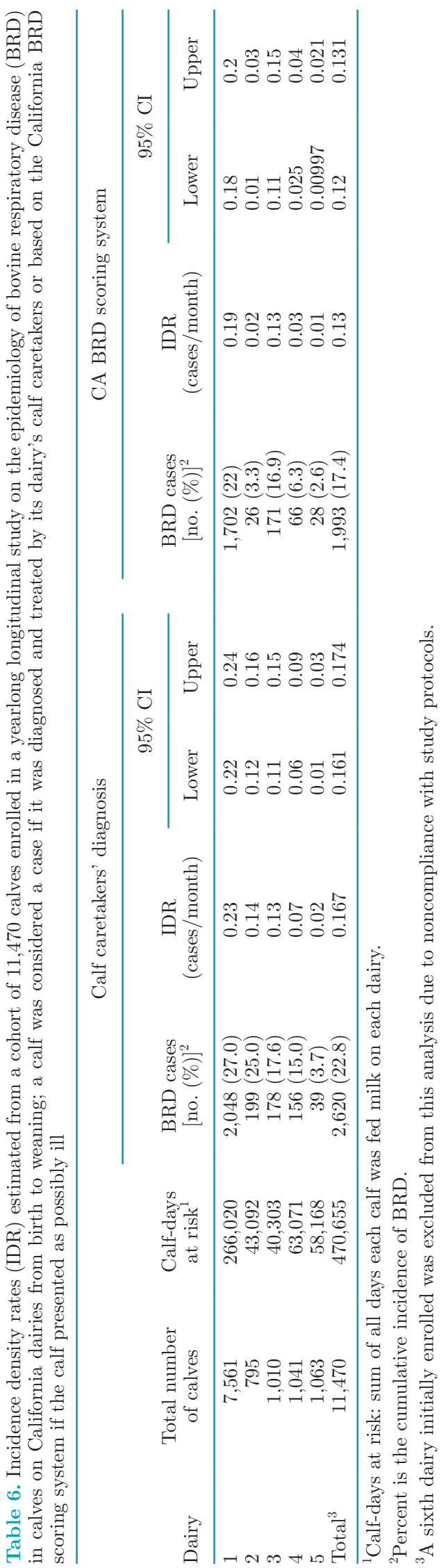


Table 7. Final shared survival frailty model for bovine respiratory disease in 11,300 calves followed from birth to weaning on 5 California dairies between March 2015 and July 2016

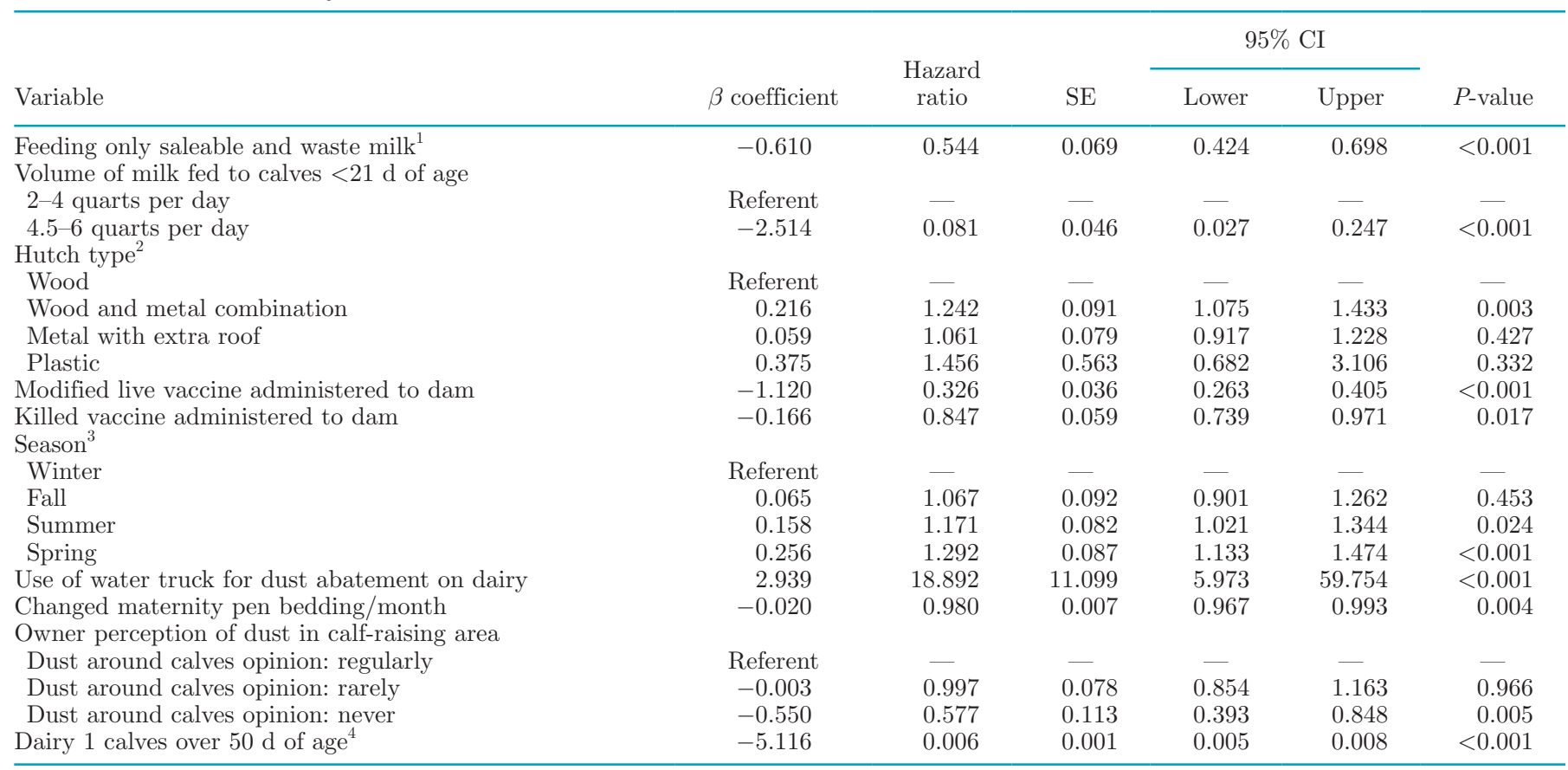

${ }^{1}$ Referent: any mixture of waste or saleable milk without milk replacer.

${ }^{2}$ Housing types included all-wood hutches (wood) as the referent, wooden hutches with metal roofs (wood and metal combination), hutches with concrete floors and incomplete walls with metal bars and a separate roof (metal with extra roof over calf hutch area), and plastic hutches (plastic).

${ }^{3}$ Season categories included the months December to February (winter), September to November (spring), June to August (summer), and March to May (spring).

${ }^{4}$ Variable was included to account for confounding in the variables for modified live vaccination administered to dam and killed vaccine administered to dam. Reference variable was all calves under $50 \mathrm{~d}$ of age on dairy 1 and all calves (any age) on the other dairies.

of the coefficient for modified live vaccination of dams by more than $20 \%$. Specifically, models with the indicator variable showed a change in the direction of the association, from modified live vaccination of dams being a risk factor for BRD to modified live vaccination having a protective effect against BRD. All crossbred calves were excluded from the model, as they were beef crosses (Limousin).

\section{DISCUSSION}

Bovine respiratory disease continues to be a significant cause of morbidity in dairy calves on California dairies, with $22.8 \%$ of the 11,470 calves in this study treated for BRD before weaning. Increasing the volume of milk fed and vaccination of pregnant dams were associated with decreased risk of BRD. Other management practices that could be modified to decrease the risk of BRD include dust abatement practices, type of milk fed to calves, and modification of calf hutch style. The current morbidity estimate (17.4\% using BRD scoring system; $22.8 \%$ using on-farm BRD diagnosis criteria) is similar to that estimated from other recent studies, such as that of Windeyer et al. (2014), in which $21.9 \%$ of all calves were diagnosed with BRD. In addition, the National Animal Health Monitoring System (NAHMS) survey found that $18.1 \%$ of calves were diagnosed with BRD in 2011 (USDA, 2012). A recent study estimated the prevalence of BRD on a convenience sample of California's Central Valley dairies and calf ranches to be $31.7 \%$ (Love et al., 2016b). The $31.7 \%$ reported prevalence from the Love et al. study may be due to the diagnosis of calves in the previous study using thoracic auscultation and ultrasound and the nested case-control study design, compared with the current estimate $(8.2 \%)$ based on calves scoring positive by observed clinical signs from several cross-sectional surveys conducted over time (Love et al., 2014). Another recent study that also did not use thoracic auscultation or ultrasound, but which sampled a larger number of dairies, estimated a BRD prevalence in 4,636 calves on 100 California dairies of $6.9 \%$ (SE 0.7; Karle et al., 2019)

A significant finding of the current study is the association between seasons in California and BRD 
hazard. Calves had the lowest hazard of BRD during the winter and fall, with highest significant hazard in the spring and summer (Table 7). In contrast, a crosssectional study based on more than 4,000 calves on 100 California dairies found that calves had higher odds of BRD during the fall than in the spring (Maier et al., 2019). The majority of studies performed in the eastern United States and Canada have found that winter is the season with the highest risk of $\mathrm{BRD}$, which may be due to the practice of collective housing indoors and lack of proper ventilation (Waltner-Toews et al., 1986). The implication of the differences in seasonal incidence of BRD between the western and eastern United States is that California dairy producers should be aggressively controlling BRD in high-risk months and during periods of large differences in daily minimum and maximum temperatures, by implementing preventative management practices.

The current study found that calf hutches made of a combination of metal and wood (a corrugated metal roof attached to wooden walls) were significantly associated with a $24 \%$ increased hazard of preweaning BRD compared with hutches made only of wood, with plastic hutches not varying significantly from onlywood hutches. The higher risk of BRD among calves housed in metal and wood combination hutches may be because a metal roof is a better heat conductor than wood, which may lead to calves experiencing elevated temperatures for longer duration during the hot California summers. The amount of energy required to maintain homeostasis in the hotter hutches may strain calves' immune system. Further research should be performed, investigating the temperature fluctuations and variations associated with different hutch types, to ascertain whether temperatures vary more in hutches with metal construction versus those made solely of wood or plastic.

A significant finding related to the amount of milk fed was that feeding over $3.8 \mathrm{~L}$ per day to calves under $21 \mathrm{~d}$ of age decreased the hazard of BRD by $92 \%$ (Table 7). Such a decrease in BRD hazard may be an indication that feeding calves less than $3.8 \mathrm{~L}$ per day may not fulfill their nutrient requirements for metabolic maintenance, growth, and immune system function. A study examining metabolic maintenance requirements for calves hypothesized that calves fed milk over the amount required for maintenance would have stronger immune systems (Drackley, 2008), which could explain the decrease in BRD hazard associated with increased volume of milk fed. The volume of milk fed on all dairies in this study exceeded the 2.5-L per day benchmark deemed necessary for metabolic maintenance (Drackley, 2008). However, the metabolic maintenance study was performed under thermoneutral conditions and does not account for the extra volume of milk necessary to meet metabolic maintenance requirements when calves are under thermoregulatory stress due to extremely hot or cold temperatures. Calves fed lower milk volumes may not receive enough nutrients to fulfill their maintenance requirements during days with large temperature fluctuations.

The frailty model results also identified that calves fed only saleable and waste milk had a lower hazard, a $46 \%$ reduction, compared with calves fed waste or saleable milk supplemented with milk replacer. Another study concluded that calves fed milk replacer had a higher risk of morbidity than calves fed pasteurized waste milk (Godden et al., 2005). Possible explanations for these findings might be that milk replacer was not being mixed properly, that it might be diluted, or that it simply does not contain enough nutrients to adequately support calves' growth and immune system health compared with whole milk either in the form of saleable or waste milk. The current study did not verify the nutrient composition of milk fed to the enrolled calves, which is a limitation to the study findings. Future studies should address this by collecting and testing samples of the milk fed at regular intervals.

The management variable with the highest hazard ratio (18.9) in the frailty model was for producerreported use of water trucks to spray roads near the calf-raising area for dust abatement (no water truck use for dust abatement being the referent). In contrast, previous studies found that dust abatement can help prevent BRD, as dust particles can significantly impair the respiratory tract defense mechanisms (Callan and Garry, 2002). One possible explanation for this seemingly contradictory result is that trucks traveling near calf-raising areas may generate additional dust, which may offset the potential benefits of spraying water to control dust.

Producer reports of dust in the calf-raising area were significantly associated with BRD, complementing the significant association between dust abatement and a higher BRD hazard. In contrast, the dairies that reported that dust was never observed had a $42 \%$ decrease in BRD hazard. Previous studies have correlated dust with increased risk for BRD because dust can be an inflammatory agent, which can increase calves' susceptibility to BRD pathogens (Callan and Garry, 2002). Dust particles impair the immune system and the respiratory tract's defense mechanisms by increasing phagocytic activity in the alveolar space, as well as impairing mucociliary clearance. Dust particles can also absorb and carry ammonia deeper into alveoli than it would travel unassisted (Hillman et al., 1992). More 
research into alternative dust abatement strategies for dairies with excessive amounts of dust in the calf-raising area is needed, to reduce the risk of BRD.

Administration of modified live and killed vaccines against BRD pathogens to preparturient cows was associated with a decrease in hazard of BRD in calves born to vaccinated dams. The current study did not attempt to compare the specific vaccines used, as that was not our objective. Calves born to dams vaccinated with a modified live vaccine were $67 \%$ less likely to develop $B R D$, and calves born to dams vaccinated with a killed vaccine were $15 \%$ less likely to develop BRD. Our findings are supported by previous studies, which found that calves born to cows vaccinated against infectious bovine rhinotracheitis and Pasteurella haemolytica, and fed colostrum from their individual dams within $8 \mathrm{~h}$ of birth, tested seropositive for these pathogens, as opposed to calves born to unvaccinated cows, indicating that vaccination of dams does have an immunological effect on calves (Hodgins and Shewen, 1994). Only 2 of the dairies in our study modified their vaccination protocols during the study period; hence, the associations between vaccination of dams using modified live or killed vaccines and BRD morbidity should be interpreted with caution. Nevertheless, the 2 dairies that changed protocols contributed $70 \%$ of the study calves. Given this sample size and the significant association between vaccination of dams and risk of BRD for modified live and killed vaccines, and findings from previous studies, it is probable that vaccination of dams does have a protective effect against BRD in preweaned calves through colostrum intake.

The current study found an association between increased frequency of maternity pen bedding changes per month and decrease in BRD risk in calves born in the same maternity pens. The likely explanation for the decrease in BRD incidence with increase in pen cleanings is reduction in the potential concentration of pathogens to which the calves are exposed during birth and immediately afterward. Previous studies have focused on individual versus group calving pens, including a study that found that calves born in individual pens had lower odds of BRD than did calves born in group pens, which fits with the theory presented above, in that individual pens should have a lower pathogen load (Svensson et al., 2003).

Our study was limited by reliance on calf caretakers to diagnose and accurately report BRD cases and clinical signs. The kappa statistic calculated, comparing calf caretaker records with clinical signs, was 0.83 and indicated excellent agreement between diagnoses using the California BRD scoring system and calf caretaker diagnoses (Fleiss et al., 2003). However, this statistic may not be an accurate representation, because most of the IDR calculated using the SSD were lower than the IDR based on calf caretaker diagnoses, which may, in turn, be due to false positives (caretakers treating calves that aren't sick) or detecting BRD cases early before calves display the full range of symptoms. Despite the limitation of relying on calf caretakers for BRD diagnosis, previous longitudinal studies with similarly large cohorts also relied on dairy staff to correctly diagnose calves with BRD (Sivula et al., 1996; Windeyer et al., 2014). In our approach, we attempted to mitigate this limitation through regular visits to the study dairy to confirm that the calf health staff continued to follow the study protocol. Indeed, this measure resulted in the exclusion of one study dairy due to lack of compliance. Another limitation of the current study was that calf health staff only recorded clinical signs on a treatment card when they diagnosed and treated a calf. A calf with a single clinical sign (for example, nasal discharge) might not have been considered a BRD case by calf caretakers, and as a result clinical signs were not recorded, limiting the kappa agreement. Cases that met California BRD scoring system case criteria but not BRD criteria of caretakers were not recorded by the calf health staff, and thus were not included in the agreement analysis. An improvement on this study design would be for dairy staff to record clinical signs observed for all calves, including those that do not meet caretakers' criteria for diagnosis of BRD.

Our study findings are also limited by the fact that only 5 dairies were used, primarily because of limitations on time and the difficulty of ensuring compliance in record keeping on the dairies when conducting longitudinal studies. Such a limitation, along with the biweekly visits to all enrolled dairies, excluded addition of more study herds. As a result, the dairies that contributed data to this study may not be representative of all California dairies and could have introduced a bias toward dairies with better record keeping for calves. This may also have indirectly biased the sample size toward dairies that do more complete and thorough calf care and management.

\section{CONCLUSIONS}

The current study followed more than 11,000 calves, one of the largest cohorts of calves studied for BRD, and provided insight into risk factors for BRD in preweaned dairy calves that are unique to California. On dairies with similar management practices to those of the study herds, several preventative measures can be implemented by managers to reduce the incidence of BRD. On dairies with high BRD incidence, herd man- 
agers should consider feeding calves more than $3.8 \mathrm{~L}$ of whole milk, commonly available on California dairies as saleable milk, although proper pasteurization of waste milk should reduce the microbial content and make this a second potential source of whole milk. Vaccinating dams for BRD pathogens during pregnancy and feeding their colostrum to calves, frequent changing of maternity pen bedding, and housing calves in non-metal hutch structures were each found to reduce the risk of $\mathrm{BRD}$ in preweaned calves. Data collected in this study suggest that implementing these changes to management practices has the potential to decrease the incidence of BRD in preweaned dairy calves in California. Such information can be applied to similar dairies in climates comparable to California's.

\section{ACKNOWLEDGMENTS}

This study was funded by competitive grant \#1753, "Risk assessment, welfare analysis, and extension education for dairy calf respiratory disease management in California" (S. Aly, principal investigator), provided by the University of California Division of Agriculture and Natural Resources, and the USDA National Institute of Food and Agriculture, Animal Health Project CALV-AH-360. The authors thank the veterinary practitioners, dairy producers, and calf caretakers who participated in this study and all the researchers who helped collect the data.

\section{REFERENCES}

Akaike, H. 1998. A new look at the statistical model identification. Pages 215-222 in Selected Papers of Hirotugu Akaike. E. Parzen, K. Tanabe, and G. Kitagawa, ed. Springer, New York, NY.

Callan, R. J., and F. B. Garry. 2002. Biosecurity and bovine respiratory disease. Vet. Clin. North Am. Food Anim. Pract. 18:57-77.

CDFA (California Department of Food and Agriculture). 2016. California Dairy Statistics Annual: 2015 Annual Data. D. M. B. CDFA Division of Marketing Services, ed. CDFA, Sacramento, CA.

Chigerwe, M., J. V. Hagey, and S. S. Aly. 2015. Determination of neonatal serum immunoglobulin $\mathrm{G}$ concentrations associated with mortality during the first 4 months of life in dairy heifer calves. J. Dairy Res. 82:400-406.

Cohen, J. 1960. A coefficient of agreement for nominal scales. Educ. Psychol. Meas. 20:37-46.

Curtis, C. R., H. N. Erb, and M. E. White. 1988. Descriptive epidemiology of calfhood morbidity and mortality in New York Holstein herds. Prev. Vet. Med. 5:293-307.

Drackley, J. K. 2008. Calf nutrition from birth to breeding. Vet. Clin. North Am. Food Anim. Pract. 24:55-86.

Dubrovsky, S. A., A. L. Van Eenennaam, B. M. Karle, P. V. Rossitto, T. W. Lehenbauer, and S. S. Aly. 2019. Bovine respiratory disease (BRD) cause-specific and overall mortality in preweaned calves on California dairies: The BRD 10K study. J. Dairy Sci. 102:73207328. https://doi.org/10.3168/jds.2018-15463.

Fleiss, J. L., B. Levin, and M. C. Paik. 2003. Statistical Methods for Rates and Proportions. J. Wiley, Hoboken, NJ.
Gardner, I. A., D. W. Hird, W. W. Utterback, C. Danayeelmi, B. R. Heron, K. H. Christiansen, and W. M. Sischo. 1990. Mortality, morbidity, case-fatality, and culling rates for California dairy cattle as evaluated by the national animal health monitoring system, 1986-87. Prev. Vet. Med. 8:157-170.

Godden, S. M., J. P. Fetrow, J. M. Feirtag, L. R. Green, and S. J. Wells. 2005. Economic analysis of feeding pasteurized nonsaleable milk versus conventional milk replacer to dairy calves. J. Am. Vet. Med. Assoc. 226:1547-1554

Greene, W. H. 2012. Econometric Analysis. 7th ed. Prentice Hall, Upper Saddle River, NJ.

Hillman, P., K. Gebremedhin, and R. Warner. 1992. Ventilation system to minimize airborne bacteria, dust, humidity, and ammonia in calf nurseries. J. Dairy Sci. 75:1305-1312.

Hodgins, D. C., and P. E. Shewen. 1994. Passive immunity to Pasteurella haemolytica A1 in dairy calves: Effects on preparturient vaccination of the dams. Can. J. Vet. Res. 58:31-35.

Karle, B. M., G. U. Maier, W. J. Love, S. A. Dubrovsky, D. R. Williams, R. J. Anderson, A. L. V. Eenennaam, T. W. Lehenbauer, and S. S. Aly. 2019. Regional management practices and prevalence of bovine respiratory disease in California's preweaned dairy calves. J. Dairy Sci. 102:7583-7596. https://doi.org/10.3168/jds .2018-14775.

Kleinbaum, D. G., and M. Klein. 2005. Survival Analysis: A SelfLearning Text. 2nd ed. Springer, New York, NY.

Lago, A., S. M. McGuirk, T. B. Bennett, N. B. Cook, and K. V. Nordlund. 2006. Calf respiratory disease and pen microenvironments in naturally ventilated calf barns in winter. J. Dairy Sci. 89:4014-4025.

Losinger, W. C., and A. J. Heinrichs. 1996. Management variables associated with high mortality rates attributable to respiratory tract problems in female calves prior to weaning. J. Am. Vet. Med. Assoc. 209:1756-1759.

Love, W. J., T. Lehenbauer, P. H. Kass, A. L. Van Eenennaam, and S. S. Aly. 2014. Development of a novel clinical scoring system for on-farm diagnosis of bovine respiratory disease in pre-weaned dairy calves. PeerJ. 2:e238.

Love, W. J., T. W. Lehenbauer, B. M. Karle, L. E. Hulbert, R. J. Anderson, A. L. Van Eenennaam, T. B. Farver, and S. S. Aly. 2016a. Survey of management practices related to bovine respiratory disease in preweaned calves on California dairies. J. Dairy Sci. 99:1483-1494.

Love, W. J., T. W. Lehenbauer, A. L. Van Eenennaam, C. M. Drake, P. H. Kass, T. B. Farver, and S. S. Aly. 2016b. Sensitivity and specificity of on-farm scoring systems and nasal culture to detect bovine respiratory disease complex in preweaned dairy calves. J. Vet. Diagn. Invest. 28:119-128. https://doi.org/10.1177/ 1040638715626204.

Maier, G. U., W. J. Love, B. M. Karle, S. A. Dubrovsky, D. R. Williams, J. D. Champagne, R. J. Anderson, J. D. Rowe, T. W. Lehenbauer, A. L. V. Eenennaam, and S. S. Aly. 2019. Management factors associated with bovine respiratory disease in preweaning calves on California dairies: The BRD 100 study. J. Dairy Sci. 102:7288-7305. https://doi.org/10.3168/jds.2018-14773.

Pithua, P., S. J. Wells, S. M. Godden, and E. A. Raizman. 2009. Clinical trial on type of calving pen and the risk of disease in Holstein calves during the first $90 \mathrm{~d}$ of life. Prev. Vet. Med. 89:8-15.

Schaffer, A. P., R. L. Larson, N. Cernicchiaro, G. A. Hanzlicek, S. J. Bartle, and D. U. Thomson. 2016. The association between calfhood bovine respiratory disease complex and subsequent departure from the herd, milk production, and reproduction in dairy cattle. J. Am. Vet. Med. Assoc. 248:1157-1164.

Scheaffer, R. L., W. Mendenhall III, R. Lyman Ott. 2006. Elementary Survey Sampling. 6th ed. Thomson Brooks/Cole, Belmont, CA.

Sivula, N. J., T. R. Ames, W. E. Marsh, and R. E. Werdin. 1996. Descriptive epidemiology of morbidity and mortality in Minnesota dairy heifer calves. Prev. Vet. Med. 27:155-171.

Svensson, C., K. Lundborg, U. Emanuelson, and S. O. Olsson. 2003. Morbidity in Swedish dairy calves from birth to 90 days of age and 
individual calf-level risk factors for infectious diseases. Prev. Vet. Med. 58:179-197.

USDA. 2012. Dairy Heifer Raiser, 2011. Pages 1-149. Animal and Plant Health Inspection Service, National Animal Health Monitoring System (APHIS-NAHMS), ed. United States Department of Agriculture, Fort Collins, CO.

Virtala, A. M. K., G. D. Mechor, Y. T. Grohn, H. N. Erb, and E. J. Dubovi. 1996. Epidemiologic and pathologic characteristics of respiratory tract disease in dairy heifers during the first three months of life. J. Am. Vet. Med. Assoc. 208:2035.

Waltner-Toews, D., S. W. Martin, and A. H. Meek. 1986. Dairy calf management, morbidity and mortality in Ontario Holstein herds. 2. Age and seasonal patterns. Prev. Vet. Med. 4:125-135.
Warnick, L. D., H. N. Erb, and M. E. White. 1994. The association of calfhood morbidity with first-lactation calving age and dystocia in New York Holstein herds. Kenya Vet. 18:177-179.

Warnick, L. D., H. N. Erb, and M. E. White. 1997. The relationship of calfhood morbidity with survival after calving in 25 New York Holstein herds. Prev. Vet. Med. 31:263-273.

Windeyer, M. C., K. E. Leslie, S. M. Godden, D. C. Hodgins, K. D. Lissemore, and S. J. LeBlanc. 2014. Factors associated with morbidity, mortality, and growth of dairy heifer calves up to 3 months of age. Prev. Vet. Med. 113:231-240. 